vast amount of information and many interesting contacts. It also helped develop working concepts for the simulated MCI. It is the first of many steps that must be taken to become prepared for a possible future MCI.

Keywords: education; mass casualty; preparedness; simulation; training; workshops

Prebosp Disast Metl 2005;20(2):554-s55

\section{Disaster Training Competencies for Healthcare Workers}

E.B. Hsu; ${ }^{1}$ T.L. Thomas, ${ }^{2}$ D.M. Whyne, ${ }^{1}$ E.B. Bass; ${ }^{3}$

G.D. Kelen; ${ }^{1}$ G.B. Green ${ }^{1}$

1. Johns Hopkins Office of Critical Event Preparedness and Response (CEPAR), Baltimore, Maryland USA

2. Loma Linda University, Loma Linda, California USA

3. Johns Hopkins Evidence-Based Practice Center, Baltimore, Maryland USA

Introduction: Although training and education have long been accepted as an integral part of disaster preparedness, many current practices have not been developed using evidence-based methods. The need for effective training of healthcare staff at all levels and "development of standards and guidelines for education and training in the multi-disciplinary health response to major events" has been identified by the 13th World Congress on Disaster and Emergency Medicine (Melbourne) as one of its highest priorities. Systematic evidence-based methods were described to derive competencies relevant to all hospital healthcare workers and staff during disasters.

Methods: A competency was defined as a broad area of personal capability, including knowledge and skill, and the application of that knowledge and skill to the standard of performance required. Cross-cutting competencies were intended to be applicable to all hospital, healthcare workers and staff. The conceptual development of competencies incorporated an evidence-based process with the following steps: (1) systematic review of existing competencies, courses, and training objectives, peer-reviewed literature, and educational theory; (2) synthesis of new competencies; (3) expert panel review; (4) refinement of new competencies; and (5) development of terminal and enabling objectives for each competency covering requisite knowledge, attitudes, and skills. Results: Through a structured, iterative, evidence-based process, the following seven competencies were developed: (1) recognize a potentially critical event and implement initial actions; (2) apply the principles of critical event management; (3) demonstrate critical event safety principles; (4) understand the institutional emergency operations plan; (5) demonstrate effective critical event communications; (6) understand the incident command system and your role in it; and (7) demonstrate the knowledge and skills needed to fulfill your role during a critical event. For each of the cross-cutting competencies, comprehensive terminal and enabling objectives were developed.

Conclusion: Cross-cutting competencies developed through a systematic evidence-based approach serve as a paradigm for hospital, healthcare worker disaster training and education. Keywords: competencies; disaster; education; evidence-base; healthcare; hospital; incident command system; management; objectives; recognition; safety; training; workers

Prehosp Disast Med 2005;20(2):s55

\section{Free Papers Theme 19: Infectious Disease}

\author{
Attitudes, Concerns, and Knowledge of Taiwanese \\ Medical Laboratory Technologists and Students \\ Regarding HIV and AIDS \\ F.F.S. Wu, ${ }^{1}$ R. Siebers, ${ }^{2}$ J.H. Liu, ${ }^{3}$ C.Y. Horng, ${ }^{3}$ \\ Y.P. Huang, ${ }^{3}$ J.Y. Lin, ${ }^{3}$ J.Y. Tsay, ${ }^{3}$ M. J. Gu, ${ }^{3}$ \\ M.H. Huang, ${ }^{3}$ C.W. Juan ${ }^{3}$ \\ 1. Changhua Show Chwan Memorial Hospital, Taiwan \\ 2. Wellington School of Medicine and Health Sciences, New \\ Zealand \\ 3. Show Chwan Memorial Hospital, Taiwan
}

Background: The World Health Organization (WHO) estimated that in 2003 there were almost 38 million people living with Human Immunodeficiency Virus (HIV) worldwide. Medical laboratory workers are the second largest professional group with potential exposure to HIV through handling of biological specimens. Previous studies from the United States, New Zealand, the South Pacific, and Finland have shown that medical laboratory workers have many unfounded fears regarding HIV and Acquired Immune Deficiency Syndrome (AIDS) that impact their work practices and attitudes towards AIDS patients.

Objective: To describe the practices and concerns of medical laboratory technologists and medical laboratory technology students regarding HIV/AIDS in central and southern Taiwan.

Methods: Questionnaires were distributed to all medical laboratory technologists and medical laboratory technology students in four hospitals (Pingtung Christian Hospital, Show Chwan Memorial Hospital, Changhua Christian Hospital, Zhushan Show Chwan Hospital) and three medical laboratory technology schools (Chunghwa Medical Technology College, Chungtai Medical Technology College, Chung Shan Medical University) in central and southern Taiwan.

Results: Of a potential pool of 530 persons, 380 (144 students and 236 medical laboratory technologists) returned questionnaires (response rate $=72 \%$ ). There was no significant difference in response rates between students and medical laboratory technologists. Respondents were predominantly female (77\%) and $196(52 \%)$ previously had attended workshops or lectures specifically on HIV/AIDS.

More than ninety percent of the respondents always wore gloves when handling a variety of biological specimens and $63 \%$ treated all specimens as potentially HIVpositive. There was a significant association between the use of gloves and the concern about acquiring HIV/AIDS or hepatitis at work $(p<0.001)$. Respondents were concerned similarly about acquiring HIV or hepatitis $B$ in their work place ( $84 \%$ and $77 \%$, respectively). A total of 44 medical laboratory technologists were seriously considering leaving the workforce because of potential contact with AIDS patients or HIV-positive biological samples. This was, in turn, related to their training and practical HIV/AIDS knowledge. 
Conclusion: Medical laboratory technologists and students in central and southern Taiwan have a deficiency of knowledge about HIV/AIDS that may impact their attitudes. The medical laboratory profession should seek to change unfounded fears of HIV/AIDS for the betterment of the profession, their immediate families, and for patients with HIV/AIDS.

Keywords: acquired immune deficiency syndrome (AIDS); education; Human Immunodeficiency Virus (HIV); knowledge; medical laboratory technologists; Taiwan

prebosp Disast Med 2005;20(2):s55-556

Infectious Disease Preparedness after SARS-A Singapore Emergency Department's Experience L.S. Quek

National University Hospital, Singapore

It has been more than a year since severe acute respiratory syndrome (SARS) took the medical world by storm. Many lessons were learned from the outbreak, which changed the way many emergency departments manage potential infectious disease outbreaks. This poster highlights the changes and measures taken by our emergency department for future responses to such threats.

The following topics will be addressed: (1) resources for appropriate infection control system available for bioterrorism and emerging infectious diseases; (2) geographical isolation; (3) air handling and filtration system in the department; (4) command and control; (5) risk communication between patients, relatives, nursing, and medical staff; (6) patient, visitor and public information management; and (7) technology—radio frequency identification (RFID), and its application in contact tracing for patients and visitors coming to the department.

Keywords: emergency department; outbreak; preparedness; response; severe acute respiratory syndrome (SARS) Prebosp Disast Med 2005;20(2):s56

\section{The Global Epidemic Recognition and Management (GERM) Chart: A Prototype \\ P. Rega; ${ }^{1}$ K. Burkbolder-Allen, RN, MSEd; ${ }^{2}$ \\ C. Budd, RN, NREMT-P,2 M. Bisesi, PbD \\ 1. Medical College of Ohio, USA \\ 2. USA}

In the past three decades, North America has witnessed an increasing vulnerability to emerging infectious diseases. This vulnerability has been accentuated in recent years as unique threats such as Anthrax, severe acute respiratory syndrome (SARS), Norovirus, and Monkeypox have emerged. Despite a highly sophisticated healthcare environment, the public health aspects of an infectious disease epidemic remain problematic.

Old and new concepts such as quarantine, isolation, alternative care sites, surge capacity, prophylaxis, and pharmaceutical stockpiling are challenging to local experts and planners. In relation, the concepts are often difficult for infrastructure personnel, healthcare providers, and the average citizen to assimilate. To meet these challenges, the authors have developed a guidance tool for the implemen- tation of community-based planning in response to an outbreak and potential epidemic.

This guidance tool is divided into three categories outlining action plans for specific segments of the local infrastructure: Category 1-Healthcare Workers; Category 2Healthcare Facilities; and Category 3-Public Health Officials. Within each category, action plans have been developed in progressive phases and are dependent upon the location of an outbreak from a global perspective and its perceived threat to communities locally, regionally and nationally: Phase 1-Limited/No Outbreak (North America Unaffected); Phase 2-Multinational Outbreak (North America Unaffected); Phase 3-North American Outbreak (Local Community Unaffected); and Phase 4North American Outbreak (Local Community Affected).

The tool's composition not only prioritizes activities, but also enhances educational endeavors. While the tool contains action plans specific to North American communities, its general concepts, and educational aspects may be exported and personalized for use elsewhere.

Keywords: action plans; global epidemic recognition and management (GERM); North America; prototype; threats; tools Prebosp Disast Med 2005;20(2):556

\section{Examination of Continuous Aspiration of Subglottic Secretions}

\section{N. Okada $;{ }^{1}$ T. Kawashima, ${ }^{1}$ N. Ishii; ${ }^{1}$ A. Takahasbi ${ }^{1}$}

M. Okasbiro, ${ }^{1}$ T. Yosbida, ${ }^{1}$ K. Okuno ${ }^{2}$

1. Kobe University Hospital, Kobe, Japan

2. Ohara Hospital, Japan

Objective: Continuous aspiration of subglottic secretions (CASS) lately has been noted because of its effectiveness for preventing ventilator-associated pneumonia (VAP). However, the method used for aspiration has not been examined in detail, so the amount of secretion obtained with CASS was determined under several conditions.

Methods: This study was conducted at the High Care Unit (HCU) of the Kobe University and Ohara Hospitals. A total of 21 patients needing prolonged intubation with tracheotomy were studied. Three types of tracheotomy tubes were examined (Types A, B, and C). First, the amount of aspiration with CASS using the Type $A$ tube was determined during 30 days with regard to gender, size of the tracheotomy tube, respiratory condition (requiring ventilation or not), and swallowing function. Second, the amount of CASS with Type B or C tubes was determined during seven days. Continuous subglottic drainage was performed with around $45 \mathrm{mmHg}$.

Results: The average daily aspiration of the Type A tube was $47.8 \pm 50.3 \mathrm{ml}$, the most being $215.2 \pm 57.4 \mathrm{ml}$ and the least being $9.8 \pm 8.9 \mathrm{ml}$. Male patients, who were intubated using a large-sized tracheotomy tube, required mechanical ventilation, and had swallowing function, tended to have greater quantity with CASS, though no significant difference was shown. The average daily aspiration quantity of the Type B tube was $2.3 \pm 3.1 \mathrm{ml}$, and that of the Type C tube was $10.0 \pm 11.7 \mathrm{ml}$. Statistically significant differences were demonstrated between the 3 types of tracheotomy tubes. 